\title{
KOREA INSTITUTE FOR ADVANCED STUDY VALUE-ADDED GALAXY CATALOG
}

\author{
Yun-Young Choi ${ }^{1}$, Du-Hwan Han ${ }^{2}$, and Sungsoo S. Kim ${ }^{1,3}$ \\ ${ }^{1}$ Dept. of Astronomy \& Space Science, Kyung Hee University, Gyeonggi 446-701, Korea \\ E-mail : yy.choi@khu.ac.kr \\ 2 Dept. of Astronomy and Atmospheric sciences, Kyungpook National University, Daegu, 702-701, Korea \\ E-mail : duegdo13@naver.com \\ ${ }^{3}$ School of Space Research, Kyung Hee University, Gyeonggi 446-701, Korea \\ E-mail : sungsoo.kim@khu.ac.kr \\ (Received August 31, 2010; Revised September 17, 2010; Accepted September 18, 2010)
}

\begin{abstract}
We present the Korea Institute for Advanced Study Value-Added Galaxy Catalog (KIAS VAGC), a catalog of galaxies based on the Large Scale Structure (LSS) sample of New York University ValueAdded Galaxy Catalog (NYU VAGC) Data Release 7. Our catalog supplements redshifts of 10,497 galaxies with $10<r_{\mathrm{P}} \leq 17.6\left(1455\right.$ with $\left.10<r_{\mathrm{P}} \leq 14.5\right)$ to the NYU VAGC LSS sample. Redshifts from various existing catalogs such as the Updated Zwicky Catalog, the IRAS Point Source Catalog Redshift Survey, the Third Reference Catalogue of Bright Galaxies, and the Two Degree Field Galaxy Redshift Survey have been put into the NYU VAGC photometric catalog. Our supplementation significantly improves spectroscopic completeness: the area covered by the spectroscopic sample with completeness higher than $95 \%$ increases from 2.119 to 1.737 sr. Our catalog also provides morphological types of all galaxies that are determined by the automated morphology classification scheme of Park \& Choi (2005), and related parameters, together with fundamental photometry parameters supplied by the NYU VAGC. Our catalog contains matches to objects in the Max Planck for Astronomy (MPA) \& Johns Hopkins University (JHU) spectrum measurements (Data Release 7). This new catalog, the KIAS VAGC, is complementary to the NYU VAGC and MPA-JHU catalog.
\end{abstract}

Key words : galaxies: fundamental parameters — galaxies: general — galaxies: distances and redshifts - catalogs — surveys

\section{INTRODUCTION}

The Sloan Digital Sky Survey (SDSS; York et al. 2000; Stoughton et al. 2002; Abazajian et al. 2009) imaged roughly $\pi$ steradians of the Northern Galactic Cap and performed spectroscopic redshift survey of galaxies, quasars and stars by using a dedicated $2.5 \mathrm{~m}$ telescope at Apache Point Observatory. The final data release (DR7) of the SDSS is one of the largest redshift samples of Main galaxies (Strauss et al. 2002) to date, completed to $r_{\mathrm{P}}=17.6^{*}$.

For large and bright galaxies with $r_{p}>14.5$, the spectroscopic completeness is unfortunately low. This is same for objects in the regions with high surface densities. The noticeable incompleteness for bright objects is due to two effects: their large flux within the $3^{\prime \prime}$ fiber aperture, and and substructures. The bright galaxies with large fiber magnitude give rise to saturation and cross-talk in the spectrographs, and their large angular size causes them to be occasionally interpreted as two

Corresponding Author: D.-H. Han

${ }^{*} r_{\mathrm{P}}$ is a galactic extinction corrected Petrosian magnitude in $\mathrm{r}$ band. or more objects after image deblending process. For these reasons, galaxies brighter than $r_{\mathrm{P}}=14.5$ have typically been excluded in the analysis. As a result, the magnitude limit of the sample reduces the range of luminosity to be explored at a given redshift and the overall number of galaxy sample. This is unfortunate with regard to understanding internal and collective physical parameters of galaxies over the large range of luminosity.

Meanwhile, the SDSS samples suffer from another incompleteness due to mechanical constraint of the spectroscopic fiber, which prevents two fibers from being placed closer than $55^{\prime \prime}$ on a given tile, thus making it impossible to observe $\sim 10 \%$ of the SDSS targets. This fiber collision problem is expected to be more serious in denser regions.

Inspired by the need for a complete catalog in redshift, we supplement the Large Scale Structure Sample of New York University Value-Added Galaxy Catalog (NYU VAGC; Blanton et al. 2005), a catalog of local galaxies based on the SDSS DR7 by bringing redshifts from the earlier literatures for the galaxies dropped from the fiber allocation procedure and the 
bright galaxies, which have no SDSS redshift. In addition to the supplementary redshifts, our catalog provides morphological types of all galaxies and related parameters such as $g-i$ color gradient, concentration index, and $u-r$ color, which are measured by an automated galaxy classification algorithm by Park \& Choi (2005). We name this catalog Korea Institute for Advanced Study ${ }^{\dagger}$ Value-Added Galaxy Catalog (KIAS VAGC).

Section 2 describes step by step the procedures employed to create the KIAS VAGC. In Section 3 we describe the main properties of the KIAS VAGC.

\section{FROM THE NYU VAGC TO THE KIAS VAGC}

\subsection{NYU Value-Added Galaxy Catalog}

Our parent sample is the Large Scale Structure Sample (brvoido $\ddagger$ ) of the SDSS NYU VAGC which is based on the seventh, final data release of the SDSS. This sample lists redshifts of 583,946 Main galaxies (Strauss et al. 2002) with $10<r_{\mathrm{P}} \leq 17.6$ in the redshift range of $0.001<z<0.5$. Note that the brvoid0 LSS Sample does not exclude the areas with questionable photometry for the sake of contiguity.

The NYU VAGC uses an independent set of reductions of the public data, the Princeton reductions (written by D. J. Schlegel et al.) which are very similar but not identical to the reductions available on the Data Archive Server or Catalog Archive Server. The differences between these two reductions are well described in Section 1 of Blanton et al. (2005).

The advantages of the LSS sample catalog as an initial catalog to start with are listed below:

- Collision Correction: Since the catalog was made for statistics of the large-scale structure, it provides a "fixed" redshift to the galaxies having no measured redshift due to the fiber collision. The redshift of its nearest neighboring galaxy that is located within its group defined with a $55^{\prime \prime}$ linking length is assigned to the fiber collision galaxy (see Blanton et al. 2005 for the details). We call this redshift a "fixed- $z$ ". During the determination of supplementary redshift, the fixed $-z$ is given the lowest assignment priority.

The NYU VAGC provides corresponding files, collisions.nearest.fits, object_twodf.fits, object_pscz. fits, and object_rc3.fits.

- Supplementary redshift: The catalog matches all of the galaxies in the photometric catalog to the

\footnotetext{
${ }^{\dagger}$ This work was initiated at Korea Institute for Advanced Study (KIAS) and much of the preparation required for this work was carried out at the KIAS.

$\ddagger$ The detailed sample selection criteria such as flux limit, completeness, and photometric properties can be found at http://sdss.physics.nyu.edu/vagc/data/sdss/lss_letter.par and http://sdss.physics.nyu.edu/vagc/lss.html.
}

ones, not only in the SDSS spectroscopic survey but also in the IRAS Point Source Catalog Redshift Survey (PSCz; Saunders et al. 2000), the Third Reference Catalogue of Bright Galaxies (RC3; de Vaucouleurs et al. 1991), and the Two Degree Field Galaxy Redshift Survey (2dFGRS; Colless et al. 2001). We noticed that the object matching for the IRAS PSCz and RC3 catalogs is not particularly successful compared to the case for the 2dFGRS. The mean angular separations of the matches for the IRAS PSCz and RC3 cata$\operatorname{logs}$ reach 40 and $45^{\prime \prime}$, respectively, showing large differences in astrometry. When SDSS source is matched to more than one redshift from different surveys, we give different priorities for redshift assignment according to their matching accuracies.

\subsection{Updated Zwicky Catalog}

Another existing catalog we use for the objects lacking redshift information is the Updated Zwicky Cata$\log$ (UZC; Falco et al. 1999). Out of 19,367 galaxies in the UZC, 10,848 galaxies located within the survey regions of the sky defined in survey coordinate (see Fig. 1 below) are first selected. The mean angular separation between the matched objects in UZC and SDSS is $1.4^{\prime \prime}$. During a process of redshift assignment described below, we gave the highest priority to the UZC redshift next to the SDSS $z$ because of the relatively small difference in astrometry of the matched objects.

\subsection{KIAS Value-Added Galaxy Catalog}

We describe in detail the steps that we have taken from the existing catalogs to the KIAS VAGC.

1. Selection of galaxies that are not in the spectroscopic catalog (the NYU VAGC brvoido LSS sample) from the imaging catalog (the NYU VAGC object_sdss_imaging catalog):

a. Rejection of stellar sources: we separated galaxies from stars using a more conservative criterion on the difference between $r$ band PSF magnitude and model magnitude, $r_{\mathrm{PSF}}-r_{\text {model }}<0.25$, to exclude a number of stellar sources in the imaging catalog, whose star-galaxy separation cut is $0.2 \mathrm{mag}$. Note that the brvoido LSS sample excludes galaxies smaller than $0.35 \mathrm{mag}$.

b. Rejection of galaxies located outside of the tiling regions: the tiling regions are intersection of overlapping tiles with a radius of $1.49^{\circ}$ and are defined as "sectors" for the spectroscopic survey (Blanton et al. 2003). The method used in defining the tiling regions is described in Section 3.1.

d. Rejection of galaxies in errors by visual check: we performed visual check on the images of objects brighter than $r_{\mathrm{P}}=16$ in order to exclude objects in errors (such as errors in de- 


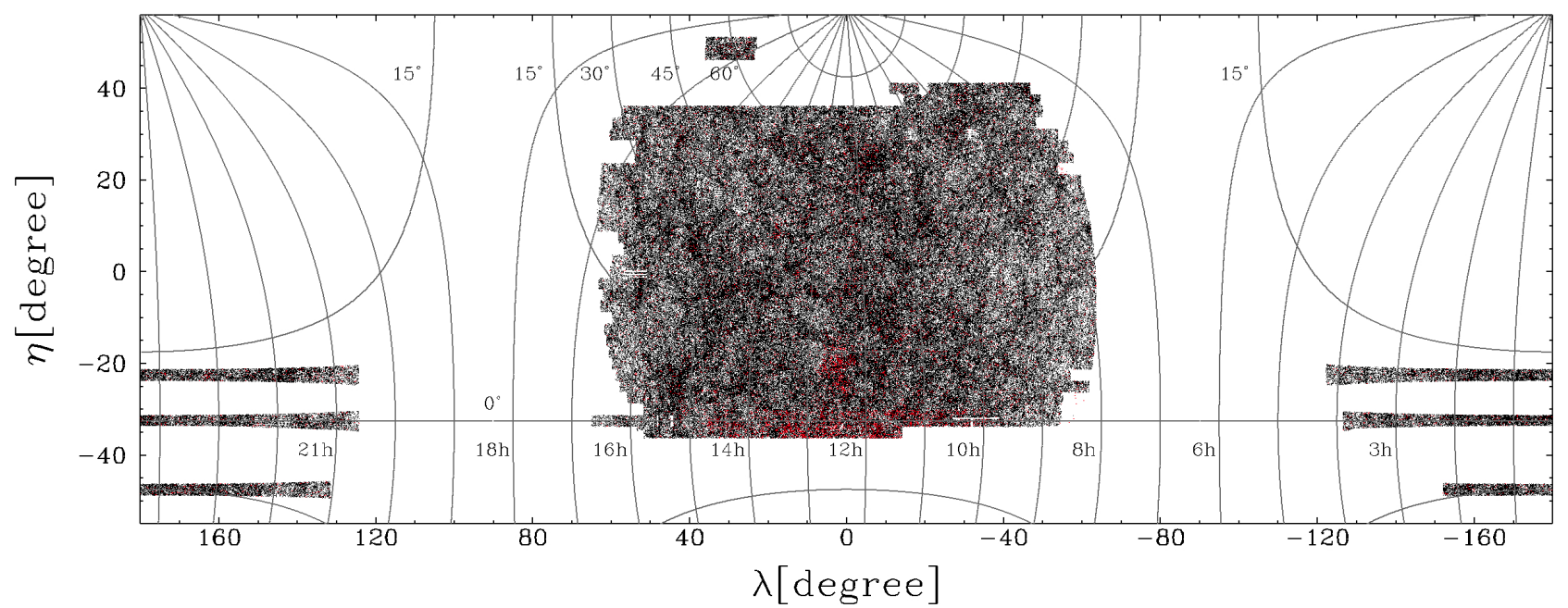

Fig. 1. - Distribution of the SDSS galaxies on the sky of the completed SDSS survey area in survey coordinate $(\lambda, \eta)$. The constant RA and DEC lines are shown as roughly vertical and horizontal curves, respectively. Black points are the galaxies in the NYU VAGC brvoid LSS catalog and the red points are those newly added in the KIAS VAGC.

blending), blank field, stellar objects, and so on. During this procedure, we found big complex galaxies or low surface brightness galaxies that were badly deblended and occasionally whose child has only spectra. In that case, we kept the spectral measurement of the child object but used the photometry information of the parent object from the imaging catalog. During the eyeball check procedure, the redshifts of badly matched objects were reset to the original value.

2. Assignment of redshift to the objects without SDSS- $z$ : When an SDSS object was matched to objects in more than one survey except SDSS, the redshift information was obtained first from UZC, then from 2dFGRS, IRAS PSCz and RC3 in order. The fixed- $z$ from the nearest neighbor was at the bottom of the assignment's priority list. About $5.6 \%$ of all galaxies have fixed- $z$.

3. Searching redshifts of the remaining galaxies in the imaging catalog using the NASA/IPAC Extragalactic Database (NED) $)^{\S}$.

Table 1 summarizes the results. Out of the galaxies in brvoid0 LSS sample which lists redshifts of 583,946 galaxies with $10<r_{\mathrm{P}} \leq 17.6,929$ erroneous objects were removed from the catalog after the visual inspection. They are mostly child objects of large galaxies, blank fields, or stars and so on. Out of the remaining 583,017 galaxies (7709 with $10<r_{\mathrm{P}} \leq 14.5$ ), 32,762 galaxies do not have spectra due to fiber collision. Out of the galaxies, 506 galaxies are cross-matched with UZC sources, 32 galaxies with RC3 sources, 1 galaxies with $2 \mathrm{dFGRS}$ source, 2 galaxies with PSCz source,

$\S$ See http://nedwww.ipac.caltech.edu and 10 galaxies with NED sources. There still remain 32,206 galaxies with fixed- $z$. For 225 galaxies that are too large in angular size and have complex substructure, the redshifts are taken from child object identified by visual inspection. We added redshift information for 10,497 galaxies with $10<r_{\mathrm{P}} \leq 17.6$ (1455 with $10<$ $\left.r_{\mathrm{P}} \leq 14.5\right)$ that are not in the brvoido LSS sample. Out of 10,497, 295 galaxies matched with UZC sources do not match with any of the object_sdss_imaging but match with imaging objects in the Catalog Archive Server (CAS) of the SDSS DR7. There are 23 UZC galaxies that do not match with any of the imaging objects both in the CAS and NYU VAGC, but are included in the KIAS VAGC.

Fig. 1 shows the distribution of SDSS galaxies in the survey area with angular selection function greater than 0, which covers 2.584 sr. Black points are the galaxies in the brvoid LSS sample and red points are those newly added in the KIAS VAGC. One can see that the added galaxies are mostly located in the high surface density regions.

All the redshift information such as the source of redshift and the angular separations of the matches are given in columns, ztype and matchdist of a file, kias_vagc_imaging_dr7. dat described in Section 3.2 below.

\section{PROPERTIES OF THE KIAS VAGC}

\subsection{Better Spectroscopic Completeness}

The resulting KIAS VAGC has significantly better spectroscopic completeness than the original NYU VAGC LSS sample. Fig. 2 shows the resulting com-

ISee htt://cas.sdss.org/ 
Table 1.

Sample statistics

\begin{tabular}{|c|c|c|}
\hline & & $\begin{array}{l}\text { Number of } \\
\text { Galaxies }\end{array}$ \\
\hline Galaxies from brvoid LSS catalog & & 583,017 \\
\hline ......... with SDSS- $z$ & {$[1]$} & 550,256 \\
\hline ... with fixed- $z$ but matched with UZC & {$[2]$} & 506 \\
\hline with fixed- $z$ but matched with 2 dFGRS & {$[3]$} & 1 \\
\hline .... with fixed- $z$ but matched with $\mathrm{PSC} z$ & {$[4]$} & 2 \\
\hline .... with fixed- $z$ but matched with RC3 & {$[5]$} & 32 \\
\hline ... with fixed- $z$ but matched with NED & {$[6]$} & 10 \\
\hline ....... still with fixed- $z$ & {$[7]$} & 32,206 \\
\hline$\ldots \ldots \ldots \ldots \ldots . . . \mathrm{w} / \mathrm{o}$ any $z$ & {$[0]$} & 5 \\
\hline Galaxies from object_sdss_imaging & & 10,497 \\
\hline .......... with SDSS- $z$ & {$[11]$} & 6,985 \\
\hline ....... matched with UZC & {$[22]$} & 959 \\
\hline ..... matched with $2 \mathrm{dFGRS}$ & {$[33]$} & 1,656 \\
\hline ..... matched with $\mathrm{PSCz}$ & {$[44]$} & 169 \\
\hline ...... matched with RC3 & [55] & 108 \\
\hline ..... matched with NED & {$[66]$} & 39 \\
\hline ...........still with fixed- $z$ & {$[77]$} & 262 \\
\hline .... Objects in CAS matched with UZC & {$[88]$} & 295 \\
\hline ... UZC Objects $\mathrm{w} / \mathrm{o}$ SDSS photometry & {$[88]$} & 23 \\
\hline
\end{tabular}

The source of the redshift is denoted as number in brackets and is given in a column ztype of the KIAS VAGC imaging catalog.

pleteness as a function of apparent $r$-band Petrosian magnitude (left panel) and redshift (right panel). The histograms show a clear change in the completeness before (dot-dashed line) and after (solid line) the redshift supplementation. The histograms in red color show the results of calculation using the sample that excludes the fixed- $z$.

We calculated the new angular selection function, a fraction of targets that successfully obtain redshifts in a sector through all the procedures described above. Figs. 3 and 4 show angular variation of the survey completeness by using the angular selection function provided by the NYU VAGC LSS data set (upper panel) and the KIAS VAGC (lower panel). To facilitate our analysis, we made four arrays of square pixels of size $0.025^{\circ} \times 0.025^{\circ}$ in the $(\lambda, \eta)$ sky coordinates that cover the analysis regions and calculated in which spherical polygon each pixel resides by using mangle routine (Hamilton \& Tegmark 2004). The spherical polygons which are occasionally called sectors were formed by the adaptive tiling algorithm (Blanton et al. 2003a) used for the SDSS spectroscopy and describe the survey geometry. The NYU VAGC provides the files containing the polygon information of the LSS sample and the geometry. We then calculated a completeness of each polygon in which each pixel resides and stored the angular selection function at each pixel of the array.
The resulting useful area within the analysis regions with non-zero selection function is 2.584 sr. Figs. 3 and 4 show that our new catalog significantly improves the angular selection functions of the survey regions particularly in high surface density regions, in the Virgo cluster region, and near the equator where the Sloan Great Wall (Gott et al. 2005) is located. After the supplementation, the survey area with the angular selection function greater than 0.97 increases from $35.9 \%$ to $50.5 \%$ of the area with the selection function greater than 0 .

The angular selection function files in binary format are provided at the KIAS VAGC Web site". The file names are sky [num] mask. data, where num denotes an area that the angular selection function covers; num $=1$ for the main area within $-65^{\circ} \leq \lambda<120^{\circ}$ and $-37^{\circ} \leq \eta<43^{\circ}$, num $=2$ for the three southern stripes (stripes 76,82 , and 86) within $\lambda>120^{\circ}$, num $=3$ for the southern stripes within $\lambda<0^{\circ}$, and num $=4$ for the Hubble Deep Field area. The areas with num $=1$ and 2 are shown in Fig. 3 and those with num $=3$ and 4 in Fig. 4. The selection function is -2 if a target is located inside the bright star mask (black dots in Figs. 3 and 4), -1 if outside the survey window geometry (grey dots in Figs. 3 and 4 ), and $>0$ if inside the survey window geometry.

\subsection{Successful Morphology Classification}

One of the most fundamental properties of galaxies is morphology. Our catalog includes morphological information for all the galaxies in it. Galaxies are classified into either early (elliptical and lenticular) or late (spiral and irregular) type, and this information is given in column morph_class of file kias_vagc_imaging_dr7.dat: morph_class $=1$ for early types, 2 for late types, and 9 for those with failed photometry.

We use the automated classification scheme developed by Park \& Choi (2005), which uses $u-r$ color, $\Delta(g-i)$ color gradient, and the inverse concentration index $c_{\text {in }}$ measured from Atlas images. Morphology information given by this scheme has been used in many studies on relation between galaxy properties and the environment (Choi et al. 2007; Park et al. 2007; Lee et al. 2008, 2010; Park, Gott, \& Choi 2008; Park \& Choi 2009; Park \& Hwang 2009; Choi, Woo, \& Park 2010; Lee et al. 2010; Han et al. 2010) and on the topology of large scale structure (Choi et al. 2010; Zhang, Springel, \& Yang 2010). Reliability and completeness of this classification reaches about 90\% (Park \& Choi 2005).

To improve the results from the automated classification, we performed visual inspection together with thirteen astronomers. We selected 83,292 galaxies that are located in the "trouble zones" where the reliability of the automated classification is relatively low. These

" http://astro.kias.re.kr/vagc/dr7/mask.htm 


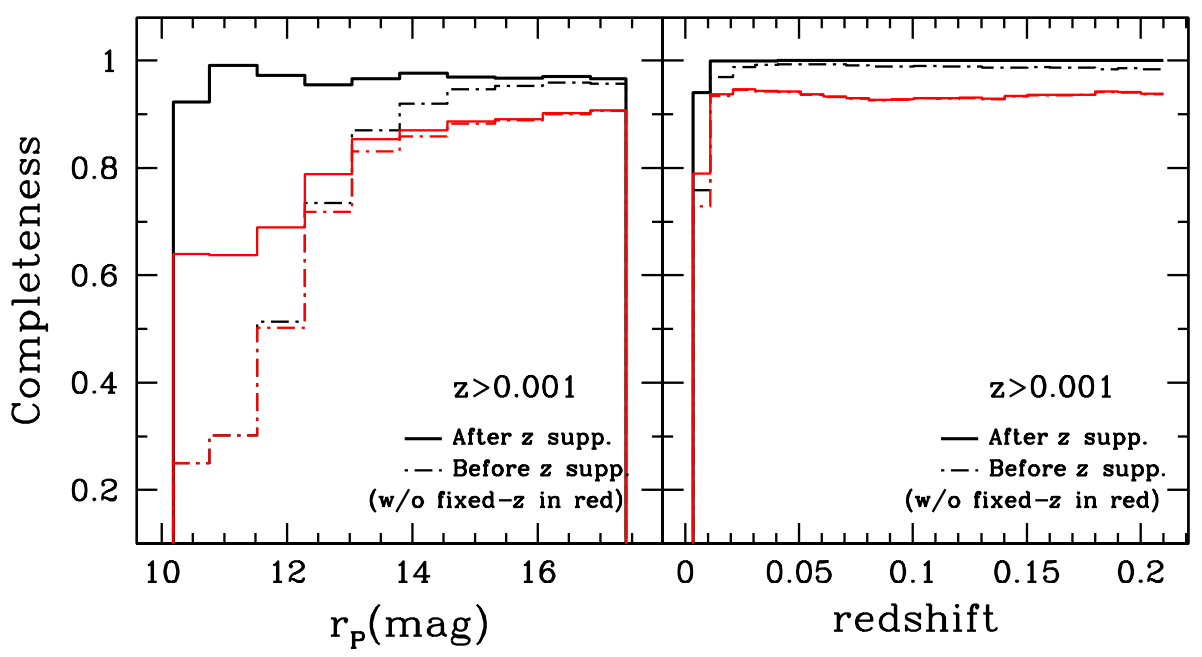

Fig. 2.- Completeness as a function of apparent $r$-band Petrosian magnitude (left panel) and redshift (right panel). Histograms in red color show the results of the calculation the using the sample that excludes the fixed- $z$.

galaxies are the ones with neighbors at very small separations ( $c_{\text {in }}$ may be inaccurate for these galaxies) or those classified as blue early types or red late types. As a result, morphology has been corrected for $7 \%$ of the inspected galaxies and some spurious objects have been removed. We kept the morphology of 5,956 galaxies in the SDSS DR4plus sample that have been already inspected by Choi et al. (2007).

Fig. 5 displays how the morphology of galaxies is classified in the $u-r$ versus (inverse) concentration index space (upper panel) and in the $u-r$ versus $\Delta(g-i)$ space (lower panel). Each morphological type is further divided into normal and abnormal classes that are designated by the following bits in column morph_type of file kias_vagc_imaging_dr7 . dat:

$>0$ : Normal late-type galaxies (morph_class=2) distributed within the parameter spaces defined by $1.8<u-r<3.5 \&-0.7<\Delta(g-i)<0.5$ or $u-r \leq 1.8 \&-0.7<\Delta(g-i)<1.0$ (blue dots in Fig. 5)

1: Normal early-type galaxies (morph_class=1) as defined above but for $u-r>2.5$ (red dots)

2: Blue early types in the trail extended out of the cluster of the typical early types (magenta dots). They are bluer $(u-r<2.5)$ and often show emission lines.

-1 : Early (orange circles) or late types (green crosses) within space $u-r \geq 3.5 \&-0.7<\Delta(g-i)<0.5$.

-2 : Early (violet circles) or late types (cyan crosses) without $\Delta(g-i)$ or $c_{\text {in }}$ measurements.

The galaxies with morph_type $=-1$ or -2 constitute only $0.76 \%$ of the entire galaxy sample. We recommend the use of galaxies with morph_type greater than or equal to 0 .
Fig. 6 shows $u-r$ color versus luminosity diagram for a volume-limited sample that includes galaxies with $M_{r} \leq-19.0$ and $0.020 \leq z \leq 0.687$. To avoid biases in color and luminosity due to dust-reddened late-type galaxies, only late-type galaxies with axis ratio greater than 0.6 are used. The red (blue) histogram shows the distribution of early (late) type galaxies in $u-r$ color and luminosity. Besides morphology, galaxies appear to be also divided into two distinct populations (red and blue sequences) that occupy two distinct loci in the color-luminosity space (Baldry et al. 2004; Bell et al. 2004). The green dashed line in the figure represents the dividing line between the red and blue sequences. Distributions of the red and blue sequences are shown in magenta and green histograms, respectively.

These morphology-related parameters are included in the KIAS VAGC, along with other fundamental photometric and spectroscopic parameters supplied by the NYU VAGC DR7. File kias_vagc_imaging_dr7.dat contains the following 36 columns, and is available on the KIAS VAGC Web site.

1. OBJECT_POSITION of the NYU VAGC. 318 galaxies with an index greater than 3,000,000 are not in the object_sdss_imaging catalog of the NYU VAGC. Out of these 318 galaxies, 295 are found in the SDSS CAS and 23 galaxies solely in the UZC.

2-4 RA, DEC (in degrees, J2000.0), and the redshift (some are negative)

5 Comoving distance in $h^{-1} \mathrm{Mpc}$, where $\Omega_{m}=0.26$ and $\Omega_{\Lambda}=0.74$ were assumed.

$6 M_{r}^{0.1}$ : Petrosian absolute magnitude at $r$-band, normalized to redshift of 0.1 . The term $+5 \log h$ is dropped, and evolution correction of $E(z)=$ 1.6( $z-0.1)$ has been made (Tegmark et al. 2004).

$7(u-r)^{0.1}$ color: rest-frame color $K$-corrected to redshift of 0.1. Galactic extinction (Schlegel et al. 

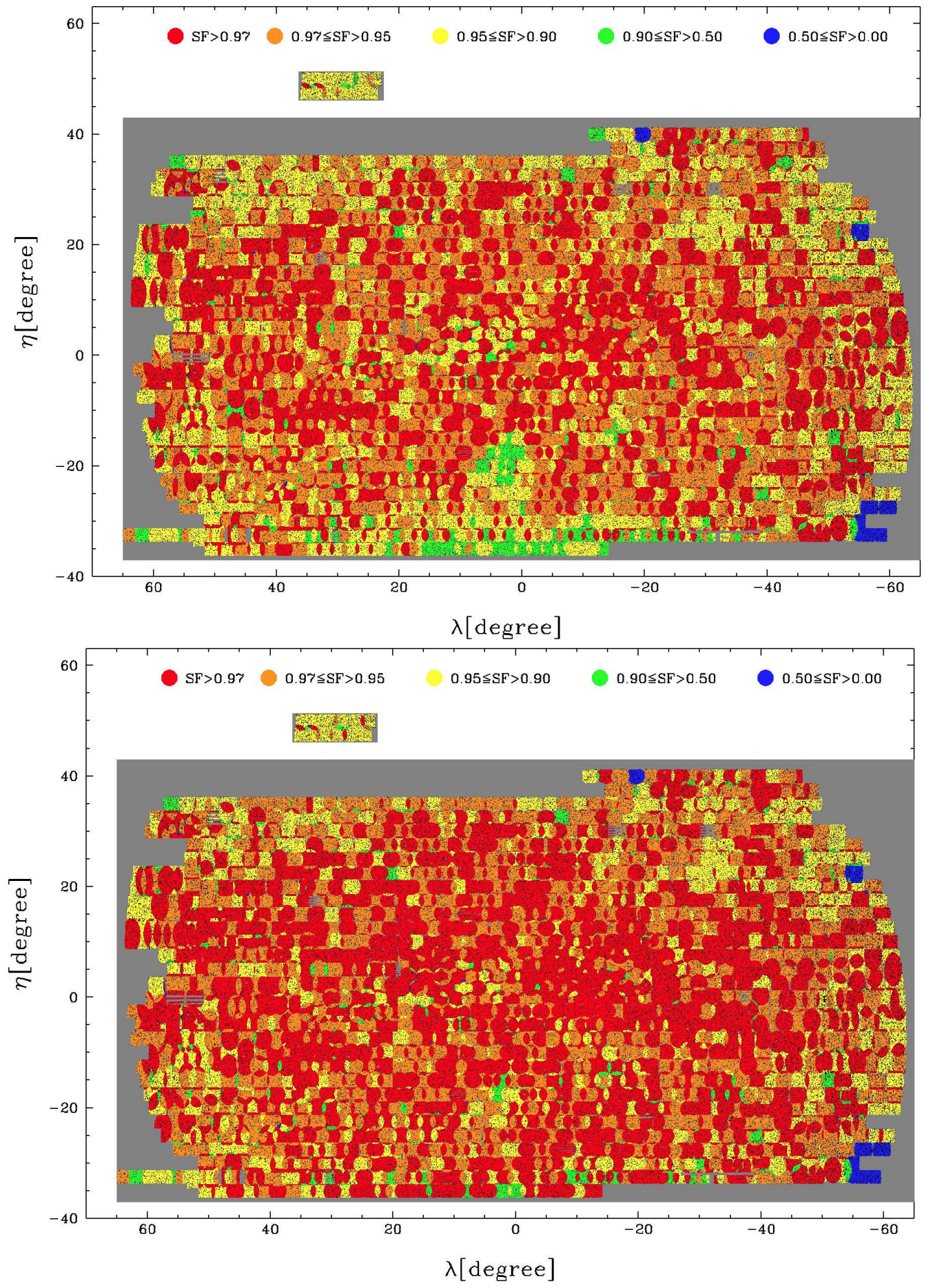

Fig. 3. - Angular selection function of the main survey and the Hubble Deep Field areas in the northern hemisphere that is adopted in our KIAS VAGC as a base catalog. The survey area is divided into five regions with different selection function ranges that are encoded by color. Note that there are many regions with selection function less than 0.90 . The angular selection functions before and after the redshift supplementation are displayed in the upper and lower panels, respectively. After the supplementation, in the main survey the area with the angular selection function greater than 0.97 increases from $36.6 \%$ to $51.6 \%$ of the area with the selection function greater than 0 ; the area with non-zero selection function is 2.331 sr. The area in black denotes bright star mask. 


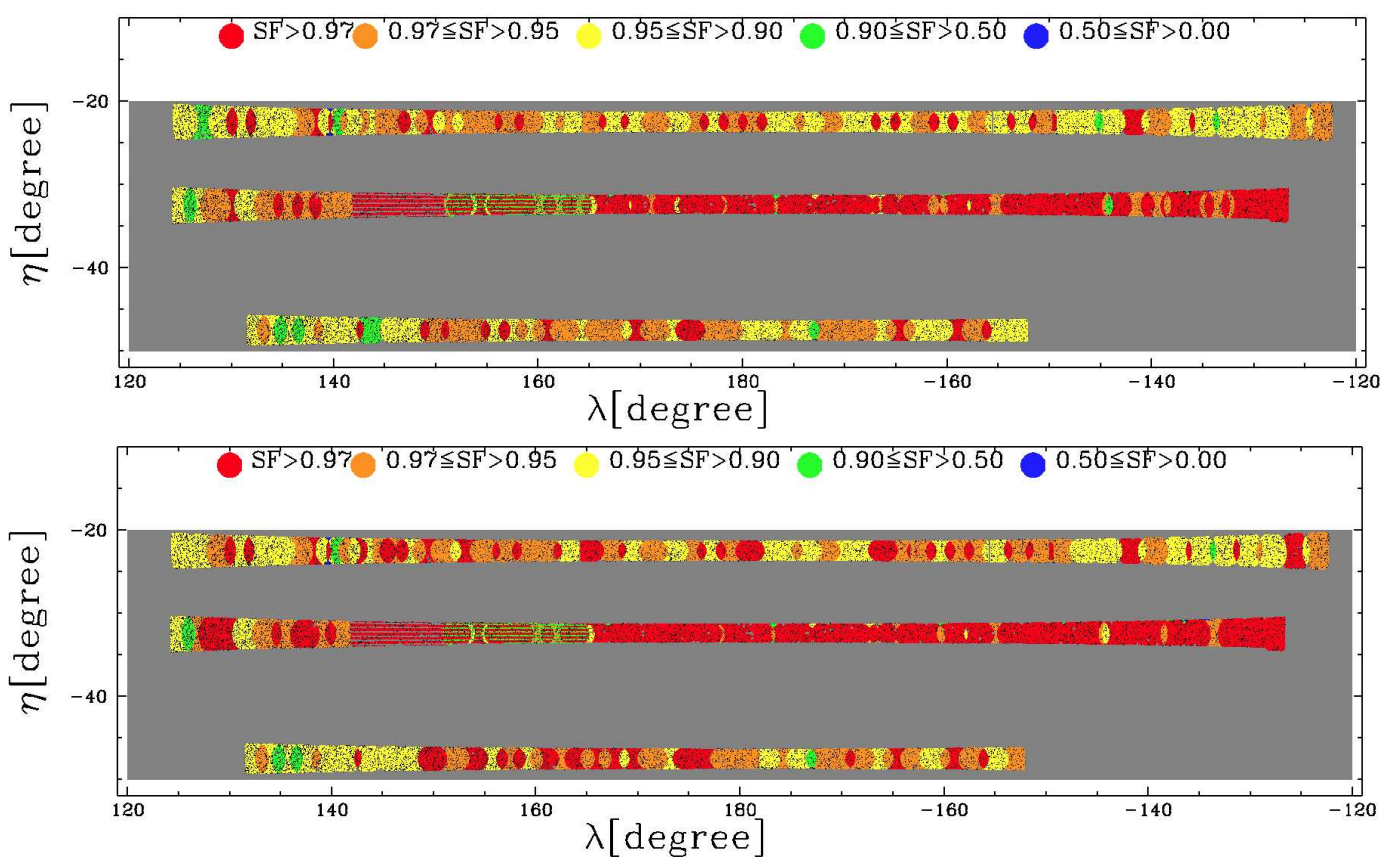

Fig. 4. - Same as Fig. 3, but showing the Angular selection function for the three southern stripes (stripes 76, 82, and 86). The angular selection functions before and after the redshift supplementation are displayed in the upper and lower panels, respectively.

1998 ) is corrected, and model magnitude is used.

8 morph_class

9 morph_type

$10 \Delta(g-i)$ : difference in $g-i$ color between the region with $R<0.5 R_{\text {Pet }}$ and the annulus with $0.5 R_{\text {Pet }}<$ $R<R_{\text {Pet }}$, where $R_{\text {Pet }}$ is the Petrosian radius at $i$-band. A negative color difference means bluer outside.

$11 c_{\mathrm{in}}$ : inverse concentration index at $i$-band with seeing correction, $c_{\mathrm{in}}=R_{50} / R_{90}$, where $R_{50}$ and $R_{90}$ are the radii from the center of a galaxy containing $50 \%$, and $90 \%$ of the Petrosian flux, respectively.

12 Galactic extinction-corrected Petrosian magnitude at $r$-band

13 Petrosian radius at $i$-band in arcsec

14 Petrosian radius at $i$-band re-calculated by using elliptical annuli in arcsec

15-16 Isophotal major and minor axis lengths at $i$-band

17 Seeing corrected isophotal $\mathrm{b} / \mathrm{a}$ axis ratio at $i$-band

18 Isophotal position angle at $i$-band in degree

19 Velocity dispersion in $\mathrm{km} \mathrm{s}^{-1}, \sigma$ : spectra with median per-pixel $S / N>10$ and $70<\sigma<420$ are recommended to be used.

20 Error in velocity dispersion: negative for invalid fit

21 Median per-pixel signal-to-noise ratio
22-23 de Vaucouleurs radius (in arcsec) and b/a axis ratio at $i$-band

24-25 Exponential radius (in arcsec) and b/a axis ratio at $i$-band

26 morph_err: -9 if objects are erroneous in deblending, blank field, stellar objects, etc. Do not use objects with morph_err $=-9$.

27 Galactic extinction-corrected $r$-band model magnitude

$28(g-r)^{0.1}$ color: a rest-frame color $K$-corrected to redshift of 0.1 .

29-31 Galactic extinction-corrected de Vaucouleurs magnitude at $g, r$, and $i$-band

32-34 Galactic extinction-corrected exponential magnitude at $g, r$, and $i$-band

35 ztype: Source catalog of the redshift. See Table 1.

36 matchdist: Angular separation between the matched objects in degrees. For 23 UZC galaxies without SDSS photometry, matchdist is set to -9 .

There is an important caveat when using Petrosian magnitudes of extended and bright objects with $r_{\mathrm{P}}<$ 14.5. The SDSS photometric pipeline overestimating sky background in the vicinity of the bright galaxies can cause systematically underestimate galaxy flux. Although the effect has been considerably corrected for the SDSS DR7, Fig. 7 shows that the systematic error still remains by comparison of $B$-band magnitude and $r_{\mathrm{P}}$ magnitude of the matched objects in UZC and 


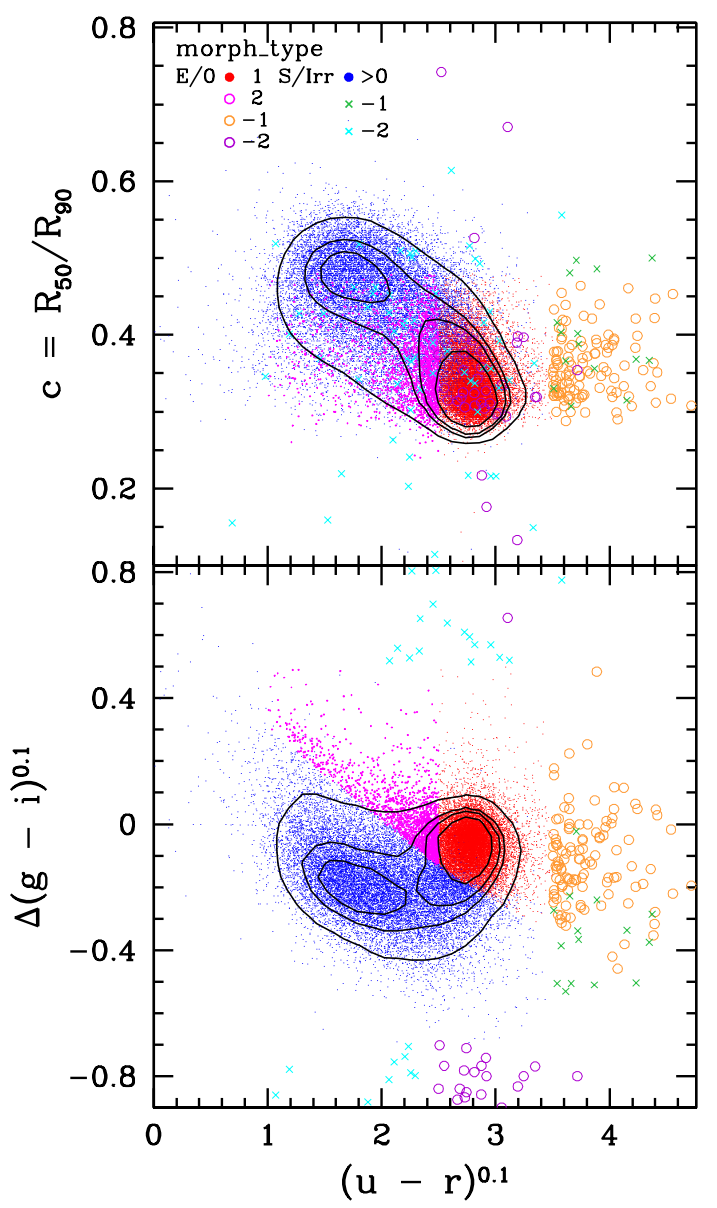

Fig. 5.- Distributions of galaxies in the $u-r$ versus the (inverse) concentration index space (upper panel) and in the $u-r$ versus $\Delta(g-i)$ (lower panel). Red, magenta, orange, and violet dots are the early types (E/S0, S0) and blue, green, and cyan crosses are the late types (S, Irr). The value of morph_type helps in selecting normal galaxies. Contours indicate the regions containing 30\%, 50\%, 70\%, and $90 \%$ of the total number of galaxies.

SDSS. For each galaxy, $r_{\mathrm{P}}$ is transformed to $B$-band magnitude using $B=g+0.33(g-r)+0.20$ from Jester et al. (2005). The secondary $y$ axis is set with $r_{\mathrm{P}}$ magnitudes corresponding to the first $y$ axis, $B$-band magnitude using $g-r=0.66$ (Fukugita et al. 1996).

\subsection{Auxiliary Catalog}

The KIAS VAGC also provides a file containing information that uniquely identifies a given spectroscopic observation, kias_vagc_spectra_dr7.dat

1 OBJECT_POSITION of the NYU VAGC DR7. The full set of spectra is in sdss_spectro_catalog.fits in the NYU-VAGC DR7, which were extracted by idlspec2d reduction code (written by D. J. Schlegel), an alternative pipeline to the official spectroscopic pipeline, spectro1d (written by M.

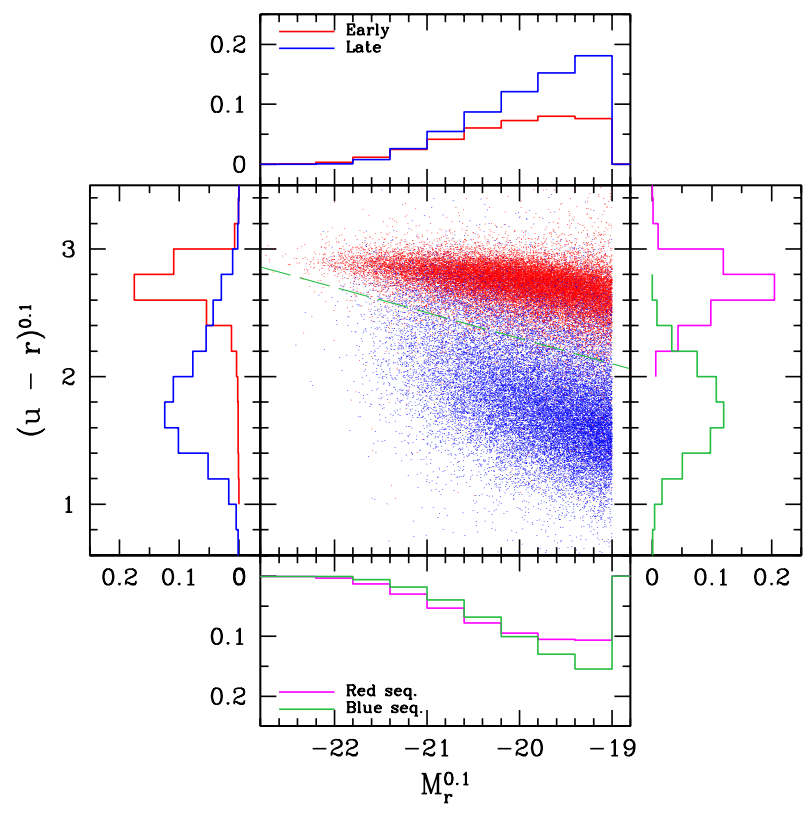

Fig. 6. $-u-r$ color vs. luminosity diagram for a volumelimited sample. Distributions of $u-r$ and luminosity are shown in the histograms; the red and blue histograms for early and late-type galaxies, and the magenta and green histograms for galaxies in red and blue sequences, respectively.

SubbaRao, M. Bernardi, and J. Frieman).

2-4 Plate number, Modified Julian Date of observation (MJD), and fiber ID (1 to 640$)$ : -1 for galaxies with redshift borrowed from the nearest neighbor, and -2 for those with redshift taken from other than SDSS.

5 MPA_POSITION: zero-based index (the first index being zero) of cross-matched objects in MPA-JHU spectrum measurement (DR7) ${ }^{* *}$.

\section{SUMMARY}

We have constructed a value-added galaxy cata$\log$ based on the SDSS final data release. Our catalog supplements redshifts not in the LSS sample of the NYU VAGC, and provides angular selection function values that are useful for the LSS study. Our catalog also provides morphological types classified by automated classification scheme of Park \& Choi (2005), along with other fundamental photometric parameters supplied by the NYU VAGC. The extended magnitude limit by adding redshifts of bright galaxies increases the overall number of galaxy sample and the range of luminosity explored at a given redshift. As a result, this advantage makes this catalog more useful for galaxy property statistics, together with the morphology information. We make this KIAS

**http://www.mpa-garching.mpg.de/SDSS/DR7/ 


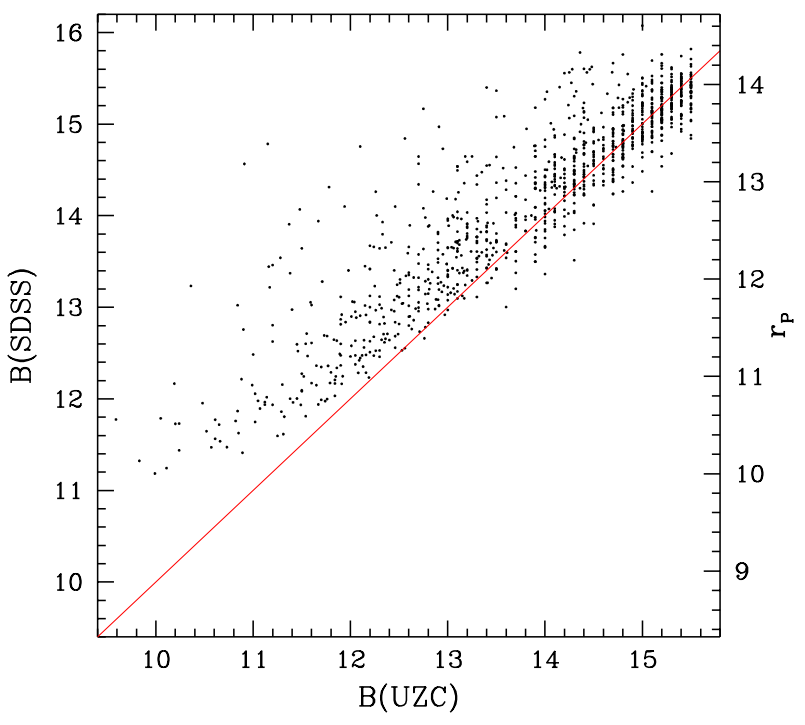

Fig. 7. - Magnitude difference between the matched objects in UZC and SDSS. The flux underestimate increases with decreasing magnitude.

value-added galaxy catalog publicly available on-line at http://astro.kias.re.kr/vagc/dr7.

\section{ACKNOWLEDGMENTS}

YYC was supported by a grant from the Kyung Hee University in 2010 (KHU-20100179). The authors thank Changbom Park for his encouragement and many valuable comments on this work. and the thirteen astronomers who joined the eyeball inspection of the galaxy morphology, Hong-Bae Ann and his two graduate students, Pravabati Chingangbam, Pascale Hibon, Ho Seong Hwang, Do-Gyun Kim, YoungRae Kim, Kwang Ho Lee, Graziano Rossi, Danny Pan, Changbom Park, and Hyunbae Park. This research has made use of the data in NYU VAGC and MPA-JHU catalog.

Funding for the SDSS and SDSS-II has been provided by the Alfred P. Sloan Foundation, the Participating Institutions, the National Science Foundation, the U.S. Department of Energy, the National Aeronautics and Space Administration, the Japanese Monbukagakusho, the Max Planck Society, and the Higher Education Funding Council for England. The SDSS Web Site is at http://www.sdss.org/.

The SDSS is managed by the Astrophysical Research Consortium for the Participating Institutions. The Participating Institutions are the American Museum of Natural History, Astrophysical Institute Potsdam, University of Basel, Cambridge University, Case Western Reserve University, University of Chicago, Drexel University, Fermilab, the Institute for Advanced Study, the Japan Participation Group, Johns Hopkins University, the Joint Institute for Nuclear Astrophysics, the Kavli Institute for Particle Astrophysics and Cosmology, the Korean Scientist Group, the Chinese Academy of Sciences (LAMOST), Los Alamos National Laboratory, the Max-Planck-Institute for Astronomy (MPIA), the Max-Planck-Institute for Astrophysics (MPA), New Mexico State University, Ohio State University, University of Pittsburgh, University of Portsmouth, Princeton University, the United States Naval Observatory, and the University of Washington.

\section{REFERENCES}

Abazajian, K. N., et al. 2009, The Seventh Data Release of the Sloan Digital Sky Survey, ApJS, 182, 543

Baldry, I. K., Glazebrook, K., Brinkmann, J., Ivezic, Z., \& Lupton, R. H. 2004, Quantifying the Bimodal Color-Magnitude Distribution of Galaxies, ApJ, 600, 681

Bell, E. F., et al. 2004, Nearly 5000 Distant Early-Type Galaxies in COMBO-17: A Red Sequence and Its Evolution since z 1, ApJ, 608, 752

Blanton, M. R., Lin, H., Lupton, R. H., Maley, F. M., Young, N., Zehavi, I., \& Loveday, J. 2003, An Efficient Targeting Strategy for Multiobject Spectrograph Surveys: the Sloan Digital Sky Survey "Tiling" Algorithm, AJ, 125, 2276

Blanton, M. R., et al. 2005, New York University ValueAdded Galaxy Catalog: A Galaxy Catalog Based on New Public Surveys, AJ, 129, 2562

Choi, Y.-Y., Park, C., \& Vogeley, M. S. 2007, Internal and Collective Properties of Galaxies in the Sloan Digital Sky Survey, ApJ, 658, 884

Choi, Y.-Y., et al. 2010, Galaxy Clustering Topology in the Sloan Digital Sky Survey Main Galaxy Sample: a Test for Galaxy Formation Models, ApJS, in publication

Choi, Y.-Y., Woo, J.-H., \& Park, C. 2009, Environmental Dependence of Active Galactic Nucleus Activity. I. The Effects of Host Galaxy, ApJ, 699, 1679

de Vaucouleurs, G., et al. 1991, Third Reference Catalogue of Bright Galxies, Volume 1-3, XII (SpringerVerlag)

Falco, E. E., et al. 1999, The Updated Zwicky Catalog (UZC), PASP, 111, 438

Fukugita, Ichikawa, T., Gunn, J. E., Doi, M., Shimasaku, K., \& Schneider, D. P., 1996, The Sloan Digital Sky Survey Photometric System, AJ, 111, 1748

Geller, M. J., \& Huchra, J. P. 1989, Mapping the Universe, Science, 246, 897

Gott, J. R., et al. 2005, A Map of the Universe, ApJ, 624,463

Han, D.-H., Park, C., Choi, Y.-Y., \& Park, M.-G. 2010, The Properties of Type $\mathrm{I}_{a}$ Supernova Host Galaxies from the Sloan Digital Sky Survey, ApJsubmitted 
Jester, S., et al. 2005, The Sloan Digital Sky Survey View of the Palomar-Green Bright Quasar Survey AJ, 130, 873

Lee, J. H., Lee, M. G., Park, C., \& Choi, Y.-Y. 2010, The Nature of the Sloan Digital Sky Survey Galaxies in Various Classes Based on Morphology, Colour and Spectral Features - II. Multi-Wavelength Properties, MNRAS, 401, 1804

Lee, J. H., Lee, M. G., Park, C., \& Choi, Y.-Y. 2008, The Nature of the Sloan Digital Sky Survey Galaxies in Various Classes Based on Morphology, Colour and Spectral Features - I. Optical properties, MNRAS, 389, 1791

Lee, G.-H., Park, C., Lee, M. G., \& Choi, Y.-Y. 2010, Dependence of Barredness of Late-type Galaxies on Galaxy Properties and Environment, ApJsubmitted

Park, C., \& Choi, Y.-Y. 2005, Morphology Segregation of Galaxies in Color-Color Gradient Space, ApJ, 635,29

Park, C., \& Choi, Y.-Y. 2009, Combined Effects of Galaxy Interactions and Large-Scale Environment on Galaxy Properties, ApJ, 691, 1828

Park, C., Gott, J. R., \& Choi, Y.-Y. 2008, Transformation of Morphology and Luminosity Classes of the SDSS Galaxies, ApJ, 674, 784

Park, C., et al. 2007, Environmental Dependence of Properties of Galaxies in the Sloan Digital Sky Survey, ApJ, 658, 898

Park, C., \& Hwang, H. S. 2009, Interactions of Galaxies in the Galaxy Cluster Environment, ApJ, 699, 1595

Saunders, W., et al. 2000, The PSCz Catalogue, MNRAS, 317, 55

Schlegel, D. J., Finkbeiner, D. P., \& Davis, M. 1998, Maps of Dust Infrared Emission for Use in Estimation of Reddening and Cosmic Microwave Background Radiation Foregrounds, ApJ, 500, 525

Stoughton, C., et al. 2002, Sloan Digital Sky Survey: Early Data Release, AJ, 123, 485

Strauss, M. A., et al. 2002, Spectroscopic Target Selection in the Sloan Digital Sky Survey: The Main Galaxy Sample, AJ, 124, 1810

York, D., et al. 2000, The Sloan Digital Sky Survey: Technical Summary, AJ, 120, 1579

Zhang, Y., Springel, V., \& Yang, X. 2010, Genus Statistics Using the Delaunay Tessellation Field Estimation Method: (I) Tests with the Millennium Simulation and the SDSS DR7, ApJsubmitted, (arXiv:1006.3768) 